\title{
Diffraction, attenuation, and source corrections for nonlinear Rayleigh wave ultrasonic measurements
}

\author{
David Torello ${ }^{\mathrm{a}, *}$, Sebastian Thiele ${ }^{\mathrm{b}}$, Katie Matlack ${ }^{\mathrm{a}}$, Jin-Yeon Kim ${ }^{\mathrm{b}}$, Jianmin \\ $\mathrm{Qu}^{\mathrm{c}}$, Laurence J. Jacobs ${ }^{\mathrm{a}, \mathrm{b}}$ \\ ${ }^{a} G W$ Woodruff School of Mechanical Engineering, Georgia Institute of Technology, Atlanta, \\ GA, 30332 \\ ${ }^{b}$ School of Civil and Environmental Engineering, Georgia Institute of Technology, Atlanta, \\ GA, 30332 \\ ${ }^{c}$ Department of Civil and Environmental Engineering, Northwestern University, Evanston, \\ IL 60208
}

\begin{abstract}
This research considers the effects of diffraction, attenuation, and the nonlinearity of generating sources on measurements of nonlinear ultrasonic Rayleigh wave propagation. A new theoretical framework for correcting measurements made with air-coupled and contact piezoelectric receivers for the aforementioned effects is provided based on analytical models and experimental considerations. A method for extracting the nonlinearity parameter $\beta_{11}$ is proposed based on a nonlinear least squares curve-fitting algorithm that is tailored for Rayleigh wave measurements. Quantitative experiments are conducted to confirm the predictions for the nonlinearity of the piezoelectric source and to demonstrate the effectiveness of the curve-fitting procedure. These experiments are conducted on aluminum 2024 and 7075 specimens and a $\beta_{11}^{7075} / \beta_{11}^{2024}$ measure of 1.363 agrees well with previous literature and earlier work. The proposed work is also applied to a set of 2205 duplex stainless steel specimens that underwent various degrees of heat-treatment over 24 hours, and the results improve upon conclusions drawn from previous analysis.
\end{abstract}

Keywords: Diffraction effects, Attenuation effects, Source Nonlinearity, Nonlinear Rayleigh waves, Nonlinear acoustics

\section{Introduction}

Nonlinear ultrasonic measurements using Rayleigh surface waves have been successfully employed to characterize material damage and microstructural changes due to a variety of failure and plastic deformation mechanisms, including fa5 tigue $^{1,2}$,cold work ${ }^{3}$, thermal aging ${ }^{4}$, and creep $^{5}$. These methods capitalize on

\footnotetext{
* Corresponding author

URL: david.torello@gmail.com (David Torello)
}

Preprint submitted to Ultrasonics

September 12, 2014 
the generation of a second harmonic component of amplitude $A_{2}$ resulting from the interrogation of a material with a monochromatic source at a fundamental frequency of amplitude $A_{1}$. These finite amplitude Rayleigh wave components are measured at multiple locations along the central axis of the ultrasonic 10 beam, providing amplitude information as a function of propagation distance. In previous works, it's been shown that the normalized second harmonic amplitude $\left(A_{2} / A_{1}^{2}\right)$ exhibits an increasing trend with propagation distance. For short ranges of propagation, the normalized second harmonic amplitude is fit well by a linear relationship, and the slope of this function is proportional to 15 the acoustic nonlinearity parameter $\beta$ of the material. It has been shown that nonlinear effects related to coupling conditions ${ }^{2}$ or system nonlinearity ${ }^{6}$ do not have an experimentally significant impact on the measurement of $\beta$ when measured over short distances because these effects exist equally at all measurement intervals. For this reason, this technique is attractive for field application due to its simplicity and robustness.

The exploitation of nonlinear stress-strain relationships to generate auxiliary signal components from monochromatic inputs has numerous applications in addition to the second harmonic generation ( $\mathrm{SHG}$ ) methods detailed in this work, including wave mixing phenomena ${ }^{7,8}$ and parametric arrays ${ }^{9,10}$. The 25 primary distinction between SHG and the latter two examples is that SHG focuses entirely on relating the harmonics generated from a monochromatic input to the nonlinearity of the material, whereas wave mixing and parametric array excitation seeks to exploit the material nonlinearity to produce output waves at sum and/or difference frequencies from multiple monochromatic inputs.

30 Despite some advantages (super-directivty of outputs and temporal isolation from inputs), the latter techniques are experimentally challenging for Rayleigh wave-based applications ${ }^{11}$.

Since the source transducer used to generate the Rayleigh wave is finite sized and directive, the radiated ultrasonic beam experiences diffraction. This mani-

35 fests as oscillatory behavior in the near field, decreasing fundamental amplitude versus propagation distance, and a nonlinearly increasing and subsequent decreasing in the second harmonic amplitude in the far field. While previous literature indicates a linear increase in second harmonic amplitude, this is not generally correct and exists primarily because the propagation distance is too 40 small in these studies to see the combined effects of diffraction and attenuation dominating second harmonic generation. This paper attempts to rectify the calculation of the nonlinearity parameter in Rayleigh wave experiments with more accurate accounting of the effects of diffraction, attenuation, and source nonlinearities.

The diffraction of a low amplitude ultrasonic beam is well understood for both three-dimensional and two-dimensional cases ${ }^{12,13,14}$, and accurate corrections for diffraction effects have been applied to the measured apparent ultrasonic wave speed and attenuation coefficient ${ }^{12}$. The diffraction of the second harmonic wave is somewhat more complicated than that of the fundamental 50 since the spreading and interference of individual rays from the source are supplemented by the spatial generation of the second harmonic waves as the funda- 
mental propagates through the nonlinear material. In the case of longitudinal wave nonlinear ultrasonic experiments, the diffraction of the nonlinear signal can generally be neglected because the propagation distance is both fixed in distance and small compared to the transducer width, which leads to minimal spreading. In addition, an integral solution has been provided for longitudinal second harmonic propagation from a piston source ${ }^{15}$, making this correction less difficult.

However, in Rayleigh wave nonlinear ultrasonic measurements, it is crucial

60 to take the diffraction effects into account because the measurements are done as a function of propagation distance and tend to extend into the far field, leading to an experimentally significant reduction in amplitude. Despite the fact that bulk measurements undergo more severe energy loss from these effects (on the order of $1 / r^{2}$ for bulk waves versus $1 / r$ for Rayleigh waves), the large prop-

65 agation distances and the fact that the measurement relies on changes in wave amplitudes versus distance means that ignoring diffraction effects will lead to significantly different values of calculated $\beta$ the further the total measurement distance is extended. For Rayleigh or Lamb waves, the derivation of a general expression for the diffraction of the second harmonic wave is intractable,

70 however Shull et al ${ }^{16}$ investigated analytically and numerically the diffraction effects in nonlinear Rayleigh waves by employing the parabolic approximation in the spectral Hamiltonian formalism ${ }^{17}$, leading to a set of partial differential equations for the fundamental and second harmonic components. Hurley et al ${ }^{18}$ compared measurements taken with a laser interferometer of a generating comb 75 transducer, characterized as a uniform finite-area source, to the theoretical results based on Shull et al and obtained a strong match between experiment and model.

In this work, we examine the source conditions of a wedge method Rayleigh wave generation scheme with finite area circular transducers in order to deter-

so mine the spatial distribution of the source amplitude as well as its harmonic content. We then apply this to a mathematical formulation based on the models proposed by Shull et al and calculate the nonlinearity parameter $\beta$ with the use of a nonlinear least squares curve-fitting algorithm in a process optimized to facilitate convergence and accuracy of the calculations in this context. This

85 process corrects for the diffraction, attenuation, and source nonlinearity terms to ensure accurate measures of $\beta$ from experimental data. Verification of this method is then provided by applying it to experimental results obtained from $\mathrm{Al}$ 2024 and Al 7075 sample measurements and also to a set of 2205 duplex stainless steel specimens that have undergone various durations of thermal aging.

\section{Background and Theory}

\subsection{Wave propagation derivations}

The following section will provide a basic overview of the derivation of the useful equations used in this research to correct for the diffraction, attenuation, and source nonlinearity of a Rayleigh wave nonlinearity measurement setup. A 

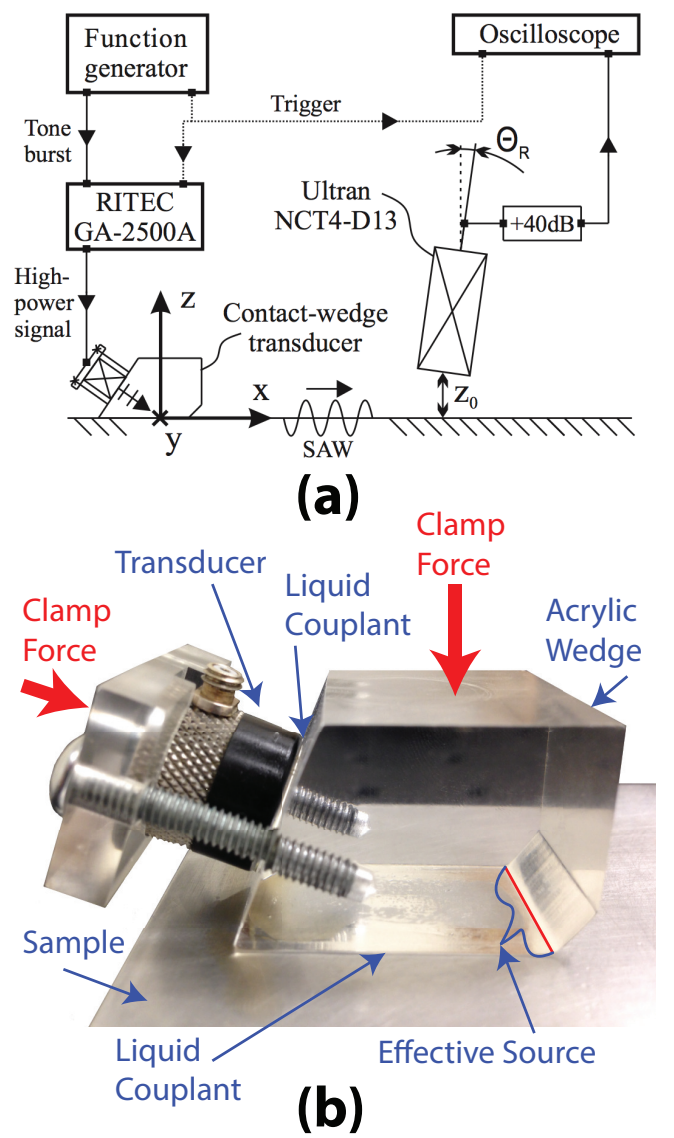

Figure 1: (a) Experimental setup schematic and theoretical framework for air-coupled transducer measurements and wedge-method generation of Rayleigh waves on the sample surface. Propagation direction of the Rayleigh wave indicated by the above arrow is positive $x$ direction, and the transverse direction along the face of the wedge is the y-direction, where $y=0$ corresponds to the center of the wedge/transducer. $z=0$ refers to the surface of the sample and becomes negative with surface depth. (b) Photograph of a contact transducer/wedge pair, noting location of the transducer, clamping forces, coupling interfaces, and the location of the effective line source (denoted by the red line).

more detailed accounting of the derivation is provided in Appendix A. If we consider a Rayleigh wave propagating along a surface in the $x$-direction of a semi-infinite half space as shown in figure 1(a), then we can describe the inplane ( $x$-axis) and out-of-plane ( $z$-axis) particle velocities at $z=0$ with the following equations ${ }^{16,17}$ :

$$
v_{x}(x, y, 0, t)=\left(\xi_{t}+\eta\right) \sum_{n=-\infty}^{\infty} v_{n}(x, y) e^{i n\left(k_{0} x-\omega_{0} t\right)}
$$


100

where the index $n$ denotes the harmonic number, $\xi_{t}=\left(1-\xi^{2}\right)^{1 / 2}, \xi_{l}=(1-$ $\left.\xi^{2}\left(c_{t}^{2}\right) /\left(c_{l}^{2}\right)\right)^{1 / 2}, \eta=-2\left(1-\xi^{2}\right)^{1 / 2} /\left(2-\xi^{2}\right), \xi=c_{R} / c_{t}, c_{l}$ is the longitudinal phase velocity, $c_{t}$ is the shear phase velocity, and $c_{R}$ is the Rayleigh phase velocity. motion for the fundamental and second harmonics of the system are ${ }^{16}$ :

$$
\begin{gathered}
\left(\frac{\partial}{\partial x}+\frac{1}{2 i k_{0}} \frac{\partial^{2}}{\partial y^{2}}+\alpha_{1}\right) v_{1}=0 \\
\left(\frac{\partial}{\partial x}+\frac{1}{4 i k_{0}} \frac{\partial^{2}}{\partial y^{2}}+\alpha_{2}\right) v_{2}=\frac{\beta_{11} k_{0}}{2 c_{R}} v_{1}^{2}
\end{gathered}
$$

where the subscripts 1 and 2 denote the fundamental and second harmonic components respectively and $\alpha_{n}$ denotes the attenuation coefficient at these frequencies.

In the above equations we see the nonlinearity parameter $\beta_{11}$, which is ultimately what we will be attempting to characterize in the later parts of this work. $\beta_{11}$ is defined by the relationship

$$
\beta_{11}=\frac{4 \mu R_{11}}{\zeta \rho c_{R}^{2}}
$$

where $\mu$ is the shear modulus, $\rho$ is the material density, $\zeta=\xi_{t}+\xi_{t}^{-1}+\eta^{2}\left(\xi_{l}+\right.$ $\left.\xi_{l}^{-1}\right)+4 \eta$, and $R_{11}$ is calculated based on the third order elastic coefficients (TEOCs) of the material and is defined by Shull et al elsewhere ${ }^{19}$. The nonlinearity parameter is theoretically calculable with knowledge of the material TEOCs, but these parameters are notoriously hard to measure empirically ${ }^{20}$

${ }_{120}$ and the parameter $\beta_{11}$ is therefore typically calculated by fitting data points with the appropriate mathematical model. In this work, the models used for curve-fitting are the solutions to equations (3) and (4) using the physical parameters of the experimental setup.

If we assume that the generating transducer combined with the acrylic wedge shown in figure 1(b) creates a Gaussian line source, the solutions to equations (3) and (4) simplify greatly. If the source function has the Gaussian form

$$
f(y, t)=v_{0,1} e^{-\left(y / a_{1}\right)^{2}} e^{-i \omega t}
$$

where $v_{0,1}$ is the peak source amplitude at $\omega$, and $a_{1}$ is the source width, then the solution for the fundamental and second harmonic components of the wave 


$$
\begin{aligned}
& v_{1}(x, y)=\frac{v_{0,1} e^{-\alpha_{1} x}}{\sqrt{1+i x / x_{0}}} \exp \left(\frac{-\left(y / a_{1}\right)^{2}}{1+i x / x_{0}}\right) \\
& v_{2}(x, y)= \frac{i \sqrt{\pi} \beta_{11} v_{0,1}^{2} k_{0}^{2} a_{1}^{2}}{4 c_{\mathrm{R}} \sqrt{i\left(\alpha_{2}-2 \alpha_{1}\right)\left(x_{0}+i x\right)}} \times \exp \left(-\alpha_{2} x-\frac{2\left(y / a_{1}\right)^{2}}{1+i x / x_{0}}\right. \\
&\left.+i\left(\alpha_{2}-2 \alpha_{1}\right) x_{0}\right) \\
& \times\left\{\operatorname{erf}\left[\sqrt{i\left(\alpha_{2}-2 \alpha_{1}\right)\left(x_{0}+i x\right)}\right]-\operatorname{erf}\left[\sqrt{i\left(\alpha_{2}-2 \alpha_{1}\right) x_{0}}\right]\right\}
\end{aligned}
$$

where $x_{0}=k_{0} a_{1}^{2} / 2$ is the Rayleigh distance and signifies the transition from near field to far field effects.

The magnitude of the fundamental particle velocity is a function of propagation distance, $x$, and is dependent on the peak source amplitude and the source width ( $v_{0,1}$ an $a_{1}$ respectively), as well as $\alpha_{1}$ (a material parameter). The magnitude of the second harmonic velocity is more complicated and additionally depends on the material parameters $\beta_{11}$ and $\alpha_{2}$. The traditional definition of the nonlinear parameter, $\beta^{\prime} \propto A_{2} / A_{1}^{2}$, is much more mathematically complicated when calculated using the solutions in equations (7) and (8) than in cases where attenuation and diffraction effects are ignored ${ }^{1}$, and the physical intuition provided by the initial definition of $\beta^{\prime}$ in these earlier works is obscured. It therefore makes more sense to fit the data to equations (7) and (8) and extract the nonlinearity parameter from the best fitting solution.

Plotting the functions for $v_{1}$ and $v_{2}$ in the $x-y$ plane and assuming an attenuation relationship of $\alpha_{n}=n^{4} \alpha_{1}$, the result shown in figure 2 generally describes the velocity profiles of the first two frequency components resulting from Rayleigh wave excitation. These plots are generated from equation (7) for the pelocity profile and equation (8) for the second harmonic velocity profile. The fundamental particle velocity decreases monotonically from the Gaussian boundary condition at $x=0$. Additionally, the second harmonic wave increases in magnitude along the central axis and then, at a large enough distance, diffraction and attenuation effects gradually overcome the effects of harmonic generation and the magnitude begins to decrease.

At $x=0$, the predicted amplitude of the second harmonic excitation is zero, according to figure 2 and equation (8). This is because the monochromatic source term defined in equation (6) naturally forces this condition to be true. In reality, piezoelectric sources generally exhibit noticeable nonlinear behavior 160 and the nonlinearity generated by the source itself, $v_{2}^{\mathrm{T}}$ (the superscript $\mathrm{T}$ denotes transducer), must be factored into the physical considerations of this problem for the calculation of $\beta_{11}$ to be accurate. A revised source term including an initial second harmonic excitation is now considered as an input to the system of equations (3) and (4)

$$
f(y, t)=v_{0,1} e^{-\left(y / a_{1}\right)^{2}} e^{-i \omega t}+v_{0,2} e^{-\left(y / a_{2}\right)^{2}} e^{-2 i \omega t}
$$



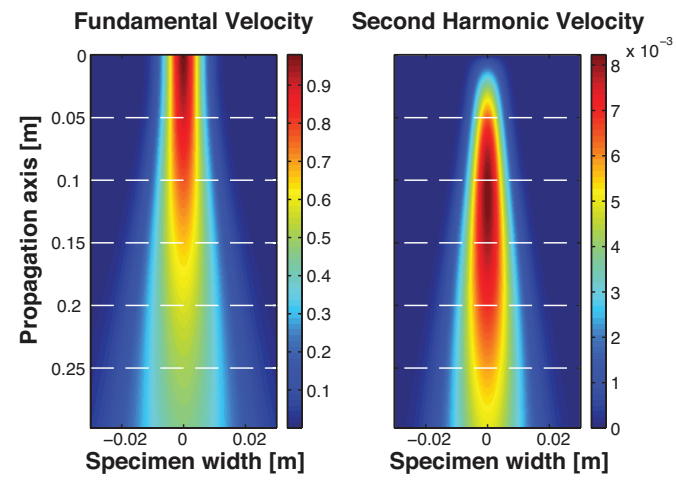

Figure 2: $x-y$ plots of the velocity distributions for Rayleigh waves excitation from a Gaussian line source from equations (7) and (8) on the left and right respectively. In these plots, the propagation axis refers to $\mathrm{x}$-direction and the specimen width refers to the y-direction from equations (7) and (8). The fundamental velocity decays monotonically from the Gaussian boundary condition at $x=0$, while the second harmonic velocity magnitude begins at zero and increases in magnitude from generation effects before reaching a maximum and decreasing due to attenuation and diffraction effects (Color online).

where $v_{0,2}$ is the peak source amplitude for the second harmonic component of the source output and $a_{2}$ is the half width of the second harmonic component.

By using this formulation of the sourcing function $f(y, t)$, the solution for the fundamental wave remains unchanged, and the solution for the second harmonic wave now can be separated into two components as follows

$$
v_{2}^{\mathrm{TOT}}=v_{2}^{\mathrm{M}}+v_{2}^{\mathrm{T}}
$$

where $v_{2}^{\mathrm{M}}$ corresponds to the nonlinearity generated in the material as the fundamental wave propagates along $x$ as described in equation (8), and $v_{2}^{\mathrm{T}}$ corresponds to the nonlinearity of the source, which takes the same form as a fundamental 175 wave propagating through the material at frequency $2 \omega$ and with properties corresponding to the material and source at this frequency. Together, the sum of these terms is equal to the total $v_{2}$, signified as $v_{2}^{\text {TOT }}$. Mathematically, the source nonlinearity is expressed in the following form:

$$
v_{2}^{\mathrm{T}}(x, y)=\frac{v_{0,2} e^{-\alpha_{2} x}}{\sqrt{1+i x / 2 x_{0}}} \exp \left(-\frac{\left(y / a_{2}\right)^{2}}{1+i x / 2 x_{0}}\right)
$$

180 Equation (11) differs from equation (7) in many ways. The attenuation of equation (7), $\alpha_{1}$, is replaced in equation (11) by $\alpha_{2}$ because the source nonlinearity occurs at $2 \omega$. Similarly, the $x_{0}$ terms in equation (7) are replaced with $2 x_{0}$ for the same reason. The final difference is the replacement of $a_{1}$ with $a_{2}$, which follows from the fact that the source has different apparent half-widths at every frequency. 
The result of decomposing the measured second harmonic signal into the framework of equation (10) is shown graphically in figure 3 for values along the propagation axis $t=0$, after converting the Rayleigh velocities $v_{2}^{\mathrm{TOT}}, v_{2}^{\mathrm{M}}$, and $v_{2}^{\mathrm{T}}$ into displacement amplitudes $A_{2}^{\mathrm{TOT}}, A_{2}^{\mathrm{M}}$, and $A_{2}^{\mathrm{T}}$ respectively. Note that because the Rayleigh velocities and the corresponding displacement amplitudes are directly related, they are referred to interchangeably in the context of this work.

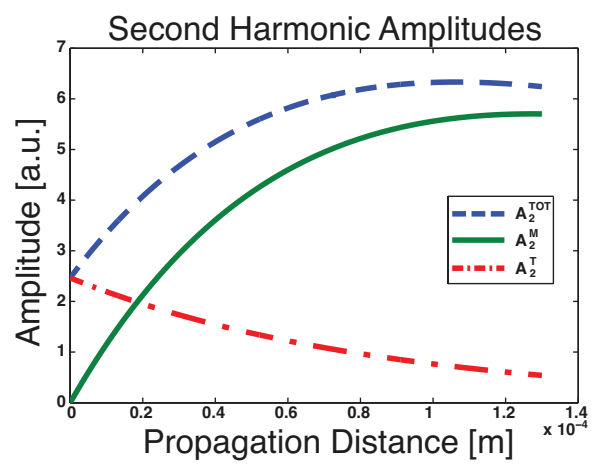

Figure 3: Values of $A_{2}^{\mathrm{TOT}}, A_{2}^{\mathrm{T}}$, and $A_{2}^{\mathrm{M}}$ predicted by theory derived in equations (8) - (10). Note that the material nonlinearity starts at zero while the actual recorded amplitude of the signal does not, which is accounted for by the peak value of transducer nonlinearity.

\subsection{Curve-fitting theory}

The fitting process employed in the calculation of $\beta_{11}$ is a nonlinear least squares curve-fitting procedure that optimizes according to the algorithm

$$
\begin{aligned}
\min _{x}\left\|v_{n}\left(\left\{v_{0, n}, \alpha_{n}, \beta_{11}\right\}, x\right)-v_{n}^{\mathrm{MEAS}}\right\|_{2}^{2} & = \\
& \min _{x} \sum_{i}\left[v_{n}\left(\left\{v_{0, n}, \alpha_{n}, \beta_{11}\right\}, x_{i}\right)-v_{n, i}^{\mathrm{MEAS}}\right]^{2}
\end{aligned}
$$

where $v_{n}$ represents the velocity functions being optimized, which in the case of the current work are $v_{1}$ in equation (7) for the fundmanetal frequency data $(n=1)$ and $v_{2}^{\mathrm{TOT}}$ in (10) for the second harmonic data $(n=2)$. Similarly, $v_{n}^{\text {MEAS }}$ represents the measured velocities at their respective frequencies. The arguments to $v_{n}$ include the values of the fitting parameters $\left\{v_{0, n}, \alpha_{n}, \beta_{11}\right\}$ and the propagation distance $x$. Note that $\beta_{11}$ is not a relevant parameter for $n=1$. The subscript $i$ appearing on the right hand side of equation (12) indicates discrete data, in this case relating to the data obtained experimentally, which implies that minimizing the cost function in terms of the experimental data solves the overall optimization problem. This method of calculating $\beta_{11}$ has been examined under different conditions and with different optimization parameters in earlier works ${ }^{18}$. 
Many of the pieces of information required for calculation of this fit are difsetup and available equipment. These parameters become curve-fitting parameters themselves in order to guarantee that they are correctly accounted for in the fitting process, however adding additional parameters can increase the likelihood of a false fit due to the introduction of local minima in the optimization

215 space. Because blind fitting to these parameters amplifies the risk of solutions at local minima, the optimizer is seeded with guesses for the input parameters that are based on theoretical and experimental insight. The effect is two-fold: the optimizer converges to a solution closest to values that make physical sense, and the optimization time is reduced. The correct starting guesses for the op220 timization parameters are either obtained from theory, comparable literature values, or experiments. The complete data-processing and curve-fitting procedures used in the experiments performed in this paper are detailed in figure 4, but germane to this discussion are the steps in figure 4(c) and (d). In figure 4(c), the fundamental amplitude is used to find values of $v_{0,1}$ and $\alpha_{1}$, which is a well-

225 defined optimization because the amplitude is almost entirely defined by $v_{0,1}$ and the shape of the data is defined by $\alpha_{1}$. These values are used later in the fitting process for the second harmonic data, which is first fit using a linear approximation over the first $n$ data points depending on the qualitative size of the "linear region" to get an initial value for $v_{0,2}$ in (d). By providing phys-

230 ically grounded and internally consistent values for these optimization steps, the final fitting in step (e), where the second harmonic information is fit using equation (10), can be assured to conform to the physics of the problem.

The process until the step shown in figure 4(c) is nearly identical to the results published on this topic in Thiele et $\mathrm{al}^{6}$ on measurements taken with air-coupled transducer setups. However, that work measures the parameter $\beta^{\prime} \propto A_{2} / A_{1}^{2}$ instead of calculating $\beta_{11}$ as described in Shull et al ${ }^{19}$.

The step shown in figure $4(\mathrm{c})$ provides the basic source strength and attenuation information from the fundamental frequency component of the filtered response, but it's important to remember that these values are affected by the 240 transfer functions of all of the components in the system (two transducers, electrical equipment, etc.). Therefore these parameters are all relative until these transfer functions are identified.

Figure 4(d) is important because it provides an initial guess for the curvefitting parameters in the step shown in part (e) and is essential for ensuring convergence and solution accuracy. The result of the nonlinear curve-fitting operation in figure $4(\mathrm{e})$ is the final value of the nonlinear source strength, the second harmonic attenuation, and ultimately $\beta_{11}$.

\section{Experimental Setup and Procedure}

\subsection{Source profile measurements}

250 3.1.1. Experimental setup

The ultrasonic generating transducer and the wedge shown in figure 1(b) combine to create an effective line source at the boundary where the wedge and 

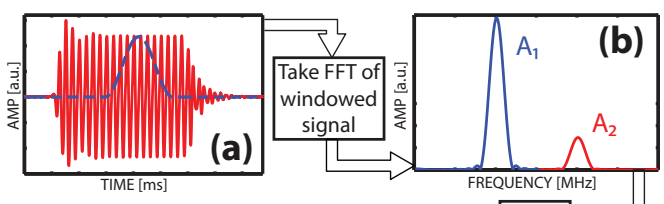

(b)

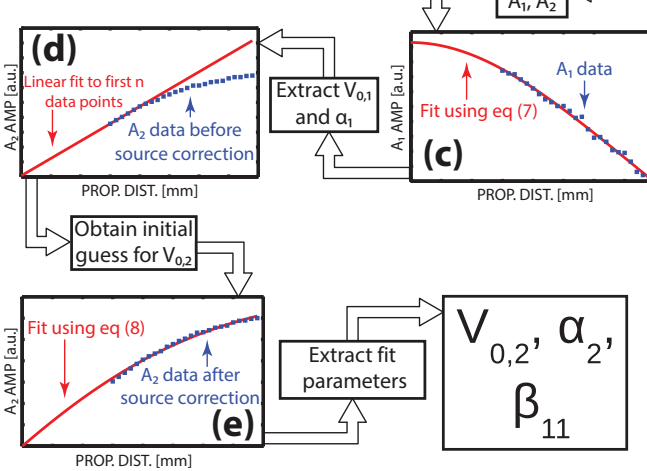

Figure 4: Diagrammatic representation of the curve-fitting procedure used to calculate the nonlinearity parameter of the measured specimen. (a) shows the signal from the receiving transducer (red) and the Hann window (dashed blue) used to filter it. This is processed with an FFT and (b) shows the frequency content at the fundamental (blue) and the second harmonic (red). The fundamental amplitude is then fit using equation (7) in (c), and the fit parameters $v_{0,1}$ and $\alpha_{1}$ are extracted and used later to fit the second harmonic data. The second harmonic data without source correction is linearly fit using the first $n$ data points (between 5 and 10) in order to get an initial value of $v_{0,2}$ to which the curve-fitting process is sensitive. This is then used in (e) to fit the $A_{2}$ data while correcting for the source nonlinearity. The resulting values of the fitting parameters are the desired results.

the sample meet. To measure the shape and magnitude of this effect, a Polytec single-point laser vibrometer was used, consisting of an OFV-551 fiber optic sensor head, an OFV-5000 controller, and a custom-built $x-y$ scanning system mounted vertically. The sample under test was a piece of 2024 aluminum. The generating setup is shown schematically in figure 5, using a half-inch Panametrics V-type transducer with a nominal center frequency of $2.25 \mathrm{MHz}$ to generate longitudinal waves in an acrylic wedge, exciting the Rayleigh waves on the specimen surface. The applied signal was generated by an Agilent 33250A signal generator and amplified by a RITEC GA-2500A RF Amplifier, which was used because of its exceptional linearity characteristics and clean output.

The signal coming directly from the laser vibrometer was amplified through a Panametrics 5072PR pulser/receiver, providing an amplification factor of $20 \mathrm{~dB}$. 265 Furthermore, the signal was discretized using a combination of a Cleverscope CS328A and a Tektronix TDS 5034B Digital Oscilloscope and later analyzed in MATLAB. 


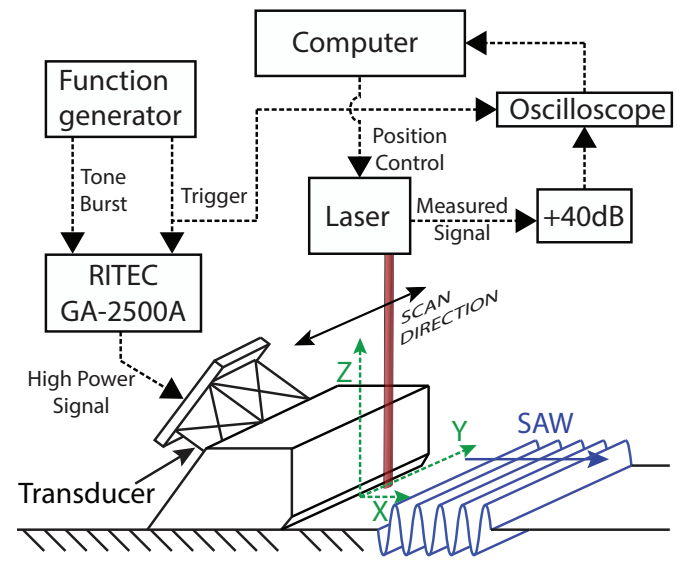

Figure 5: Laser measurement schematic showing the measurement of the effective line source at the interface between the wedge and the sample

\subsubsection{Experimental procedure}

The transducer was first applied to the wedge and clamped into position using light lubrication oil as an acoustic couplant, and this assembly was further clamped to the specimen and coupled acoustically with the same oil. Then, an input signal of $2.1 \mathrm{MHz}$ over 20 cycles with a pulse repetition rate of $20 \mathrm{~ms}$ was applied to the generating transducer, which then propagated through the acrylic wedge to the surface.

275 The resulting Rayleigh wave was measured by aligning the laser vibrometer to the front surface of the wedge such that it was as close to the contact point between the wedge and the surface as possible. The laser was scanned in the $y$-direction (along the face of the wedge), and the Cleverscope and digital oscilloscope recorded the signal, averaging over 512 cycles and sampling with a rate

280 of $250 \mathrm{MS} / \mathrm{s}$. The data was saved using a Labview script and imported into MATLAB for data processing.

\subsubsection{Data processing}

To obtain the fundamental and second harmonic frequency components of the signal, the averaged and amplified time domain data was filtered using a

285 Hann window in the steady state portion of the received signal. This effectively eliminated the ringing of the generating transducer. The signal was then processed using the MATLAB FFT algorithm and the contributions of the fundamental and second harmonics to the signal were extracted and assessed. Finally, the fundamental frequency data was fit to the corresponding frequency term in the Gaussian objective function represented by equation (9), and likewise for the second harmonic data and the corresponding term at $2 \omega$. This was performed in the optimization toolbox in MATLAB. 


\subsection{Air-coupled transducer measurements}

\subsubsection{Experimental setup and procedure}

295 The air-coupled transducer measurements were obtained with the setup depicted in figure 1. Thiele et al covers the measurement procedure in detail in a previous work on this subject ${ }^{6}$. A basic summary of the measurement follows here.

The generating system is again an acrylic wedge coupled with an ultrasonic generating transducer (Panametrics V-type, center frequency of $2.25 \mathrm{MHz}$ and $12.5 \mathrm{~mm}$ diameter), excited by an Agilent $33250 \mathrm{~A}$ signal generator amplified by a RITEC GA-2500A RF Amplifier. The input pulse shape was again a 2.1 $\mathrm{MHz}$ sine wave modulated by a rectangular window of 20 cycles with a pulse repetition rate of $20 \mathrm{~ms}$. The receiver is an Ultran NCT4-D13 12.5mm diameter

305 air-coupled transducer, amplified by a factor of $40 \mathrm{~dB}$ by the pulser/receiver and held at a distance of $3.5 \mathrm{~mm}$ from the surface of the specimen under test.

Propagation distances for this experiment were chosen between $x_{\min }=30$ $\mathrm{mm}$ and $x_{\max }=78 \mathrm{~mm}$, with the starting distance chosen primarily because of restrictions from the size of the air-coupled transducer and the assembly that houses it. Two millimeter step sizes provided adequate spatial resolution to see the major observable effects while maintaining a reasonable measurement time. The measurements were conducted first by calibrating the lateral position and angle and of the main lobe at the fundamental frequency and then the scans were performed by manually adjusting the $x-y$ position of the air-coupled transducer to maintain this line. This is very important for repeatability of the results ${ }^{21}$.

The air-coupled transducer was scanned along this centerline at a constant standoff height of $3.5 \mathrm{~mm}$ from the surface at an angle of approximately 6.5 degrees for the aluminum sample. The physical method that the air-coupled transducer uses for detection of the out-of-plane displacement of the Rayleigh 320 wave is the leakage of energy from the surface of the specimen into the air according to the predictions and theory developed by Deighton et $\mathrm{al}^{22}$ and is a consequence of Snell's Law, which predicts the transducer must be angled at $\Theta_{R}$, where

$$
\Theta_{R}=\sin ^{-1}\left(\frac{c_{a i r}}{c_{R}}\right)
$$

325 This out of plane displacement was then related back to the definition of the Rayleigh displacement by the relationship established by equation (2).

The air-coupled transducer has a nominal center frequency of $4 \mathrm{MHz}$ and an actual center frequency of $3.9 \mathrm{MHz}$. The second harmonic in this measurement system (at $4.2 \mathrm{MHz}$ ) falls within the bandwidth of the transducer. Amplification and averaging over 256 cycles resulted in an SNR of $54 \mathrm{~dB}$ for these measurements, which is enough to resolve the second harmonic data adequately. This data was recorded on the Tektronix oscilloscope and imported into MATLAB for data processing. 


\subsubsection{Data processing}

\subsection{Source nonlinearity measurements}

The results of the source nonlinearity measurements described in section 3.1.1 show that there are indeed higher harmonic components to the signal that propagates from the contact interface between the acrylic wedge and the sample source signal. Looking at the distribution along the $y$-axis at the fundamental and second harmonic frequencies $(x=0)$ gives the results shown in figure 6 .

Figure 6 suggests that the fundamental (a) and second harmonic (b) data is fit accurately by a Gaussian profile, with R-squared values of .904 and .703

375 respecitvely. The R-squared value can be interpreted as the proportion of the variation in the data that is accounted for by the model in question, so a perfect 


\section{Source Linear Response}
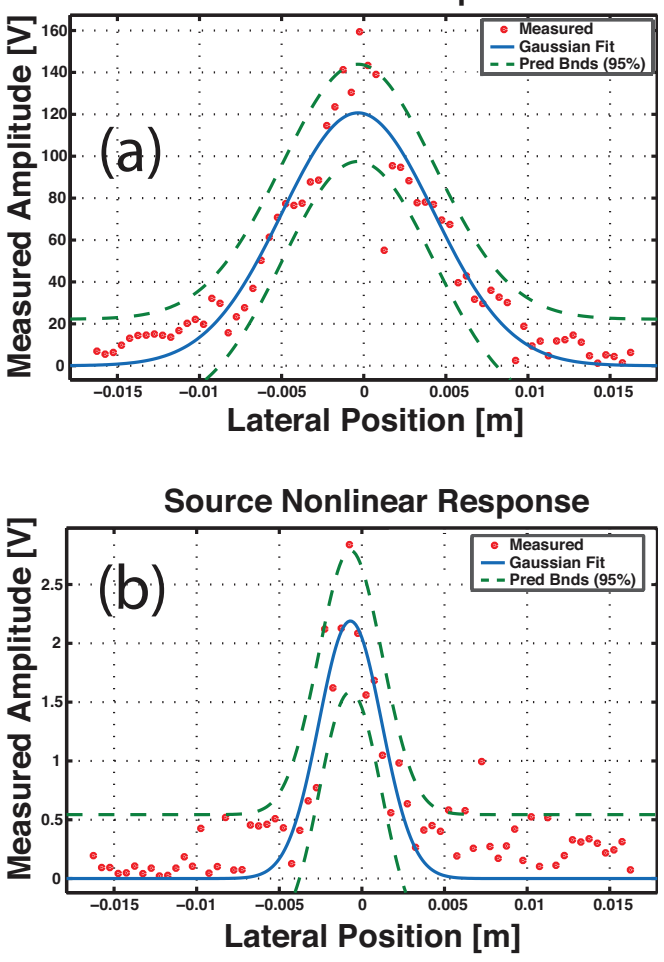

Figure 6: The response at $x=0$ from the transducer at the fundamental (a) and second harmonic (b) frequencies versus the distance from center of the wedge in the $y$-direction. Also included are the prediction bounds for a 95\% confidence interval surrounding the curve fit, which is a Gaussian in profile.

model will give a value of 1 . In this case, the fit of the fundamental source term is very high, with only roughly a ten percent variation in the data not being accounted for by a Gaussian fit, and there is high qualitative agreement.

380 The second harmonic performs slightly worse with a value of .703, but the reduction of the R-squared value can come from many sources not related to the goodness of fit. Some of these conditions present in this system are basic variances in the data acquisition at low signal amplitudes approaching the noise floor of the receiver (the second harmonic amplitudes are very small) as well as surface conditions and slight misalignment of the optics, which would affect the SNR of the measurement system. Thus a high qualitative agreement, mixed with a reasonably high $\mathrm{R}$-squared value, is confirmation that a Gaussian model accurately fits the second harmonic data.

Another observable effect is that the Gaussian beam width produced at the second harmonic is smaller than that produced at the fundamental. The fundamental beam width was measured to have a half-width, which corresponds to the radial term $a$ in the Gaussian source equation, of $6.53 \mathrm{~mm}$. This is 
slightly larger than the radius of the transducer, and is evidence that there is diffraction and perhaps second harmonic effects occurring within the wedge during generation. The second harmonic beam half-width was $2.69 \mathrm{~mm}$, which makes sense according to standard acoustic consideration ${ }^{24}$ and the graphical results observed from figure 2. Aside from confirming physical assumptions about the data, this information is important for numerical considerations and determining the values of parameters in the quasilinear solutions.

For the proposed theoretical framework for analyzing $\beta_{11}$ with nonlinear Rayleigh waves to be valid, the source must be a line source with Gaussian amplitude profile at all frequencies of interest ${ }^{16}$. The preceding analysis has demonstrated all of these conditions to be true, and thus the solutions determined in equations (7) and (8) are applicable to the experiments performed in this work, as well as any nonlinear Rayleigh wave experiment that satisfies these source conditions.

\subsection{Nonlinear ultrasound measurements}
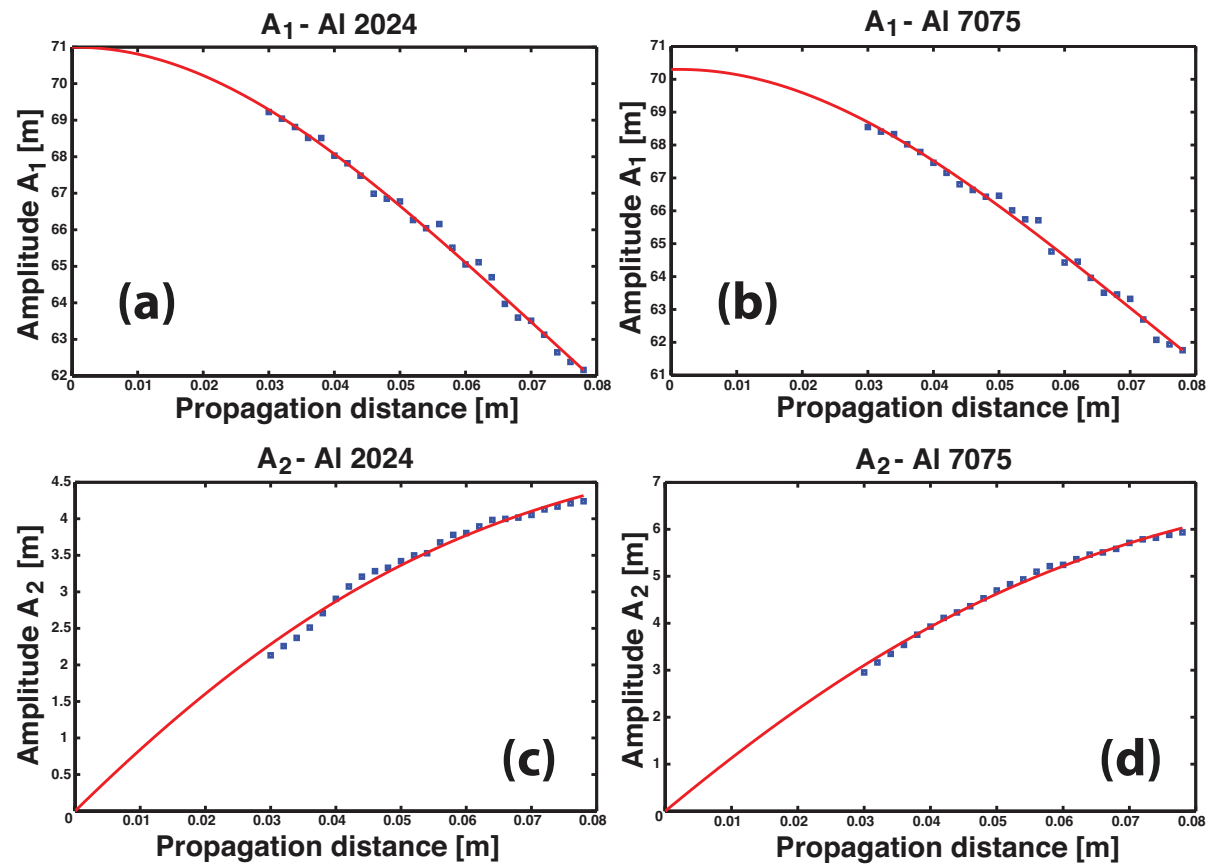

Figure 7: Nonlinear ultrasound testing results for the $\mathrm{Al} 2024$ and 7075 samples. (a) and (b) show the fundamental amplitudes for the 2024 and 7075 specimens respectively. (c) and (d) show the second harmonic amplitudes for the 2024 and 7075 specimens respectively. Data points and best-fit lines from the optimization process outlined in figure 4 are shown for each case

The results of the aluminum 2024-T351 and 7075-T651 plate measurements ${ }^{25}$ are shown in figure 7 . In these figures, the fundamental components ( $a$ and b) 
410

2024 and 7075 respectively are shown along with the results of the curve-fitting process. When interpreting these figures, it is again important to understand that fitting the values of parameters that exclusively affect the amplitude of the data will produce numerical results that are relative to transfer functions

the measurement equipment. Therefore, without precise calibration of these transfer functions, the numerical results must either be normalized or compared across specimens making sure that the source strength $v_{0,1}$ is comparable in value (which should be the case for consistent measurements regardless). In this case, the curve-fit value of $v_{0,1}$ was $9.368 \mathrm{e} 8$ [a.u.] for $\mathrm{Al} 2024$ and 9.267e 8

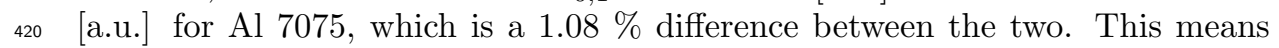
that the relative amplitudes between the two methods can be compared with confidence.

The shapes of the figures are defined by their generation and decay rates, and are thus dependent on the terms inside the exponential, radical, and error functions of equations (7) and (8). The relationships between the effects these terms have on the shape of the data versus scaling effects are quite complicated, which makes them very sensitive to change during the curve-fitting process. While the source strength values tend to converge very quickly, the terms that affect the shape of the data change dramatically and have a stronger ${ }_{430}$ influence on the quality of the fit. That being said, one of the great strengths of this curve-fitting procedure is that all of these considerations are taken care of simultaneously and automatically, and the process is repeatable and stable.

In figure $7(\mathrm{c})$ and (d), a noticeable oscillation of the data points about the prediction curve exists due to the kinematics of the manual positioning stages as they are adjusted between measurements. While these effects are worth mentioning because they appear consistently in the data sets, they do not heavily influence the results of the curve-fitting procedure.

From the process used to generate the results in figure 7, the calculated values of $\beta_{11}$ are shown in Table 1 along with results from comparable works ${ }^{26,27,25}$

Table 1: Literature and current work data for nonlinearity parameter in comparable $\mathrm{Al}$ specimens

\begin{tabular}{|c|c|c|}
\hline Data Source & Materials & $\beta_{11}^{7075} / \beta_{11}^{2024}(\max -\min )$ \\
\hline Yost et al ${ }^{26}$ & $\begin{array}{l}\mathrm{Al} 7075 \\
\mathrm{Al} 2024\end{array}$ & $1.865(2.03-1.70)$ \\
\hline Li et $a^{27}$ & $\begin{array}{l}\text { Al 7075-T551 } \\
\text { Al 2024-T4 }\end{array}$ & $1.125(1.28-0.97)$ \\
\hline Thiele et $\mathrm{al}^{25}$ & $\begin{array}{l}\mathrm{Al} 7075-\mathrm{T} 651 \\
\mathrm{Al} 2024-\mathrm{T} 351\end{array}$ & $1.675(1.85-1.50)$ \\
\hline Current work & $\begin{array}{l}\text { Al 7075-T651 } \\
\text { Al 2024-T351 }\end{array}$ & $1.363(1.52-1.25)$ \\
\hline
\end{tabular}

440

These results compare favorably to those found in literature, although it is important to note that the literature values in the cases of Yost et $\mathrm{al}^{26}$ and 
Li et $\mathrm{al}^{27}$ are for specimens of aluminum that have undergone different heat treatments and are of different chemical compositions from those tested in Thiele et $\mathrm{al}^{25}$ and the current work. However, the fact that the current work falls within the ranges obtained by the other authors speaks to the accuracy of the proposed method for the calculation of the nonlinearity parameter.

Further results are obtained from a data set borrowed from Ruiz et $\mathrm{al}^{4}$ in which heat treatment of a duplex steel sample (2205 stainless) was performed and nonlinear ultrasonic measurements taken with a contact transducer and wedge (identical to the one shown in figure 1(b)) as a receiver. This method of measuring the nonlinearity parameter is inherently less precise than methods using air-coupled transducers because of the additional interfaces between the receiving wedge to the specimen and the receiving transducer to the receiving wedge. In cases like these where the variation of the data is large, guessing which 455 points define the "linear region" of the data is highly prone to subjectivity, and avoiding this step makes results more reliable and repeatable.

By looking at the results of figure 8(a), the difference in the fitting of the calculated ratios of $A_{2} / A_{1}^{2}$ at 24 hours of heat treatment between the linear and nonlinear fitting methods is clearly shown both quantitatively and qualitatively.

${ }_{460}$ The process used to calculate the linear fit is difficult to automate or standardize because the metrics that are typically used to deduce goodness-of-fit, such as an $\mathrm{R}$-squared value, can often be misleading. If the linear fit is conducted over the entire data region, then the R-squared value would, in this case, be higher than in all other data ranges. However, this fit clearly does not follow the qualitative ${ }_{465}$ trend of the data and will become much worse with longer propagation distances in addition to demonstrating poorer accuracy in shorter propagation ranges. A linear fit to the first $n$ data points that collectively define the "linear region" will be much more accurate in short propagation ranges but will rapidly lose accuracy in the far field. This effect was discussed briefly in section 1 and is 470 primarily due to the smaller contributions of attenuation and diffraction with small propagation distances. Because standard goodness-of-fit metrics are hard to apply, the most easily conducted method of determining the "linear region" is therefor by inspection, which has obvious subjective disadvantages rooted in human error. The quantitative disadvantages, however, become glaringly 475 obvious in figure $8(\mathrm{a})$ at the propagation distance of $100 \mathrm{~mm}$, where the linear fit no longer passes through the error bars of the measured data.

The question of repeatability, consistency, and accurate accounting of acoustic considerations in the far field versus short range accuracy before attenuation and diffraction begin to dominate is answered by the nonlinear fitting method in this work, the results of which are shown as the black dash-dotted line in figure 8(a). The nonlinear fit clearly shows strong accuracy to the measured data points as well as the ability to accurately reflect the trend of the data as it enters the far field. At all points in the measurement region, the nonlinear fit passes through the error bars of the data. These advantages are present in 485 all of the measurements conducted on every specimen in the 2205 duplex stainless steel data set, and because the subjectivity of the linear fitting method is removed, the results are repeatable as well. 

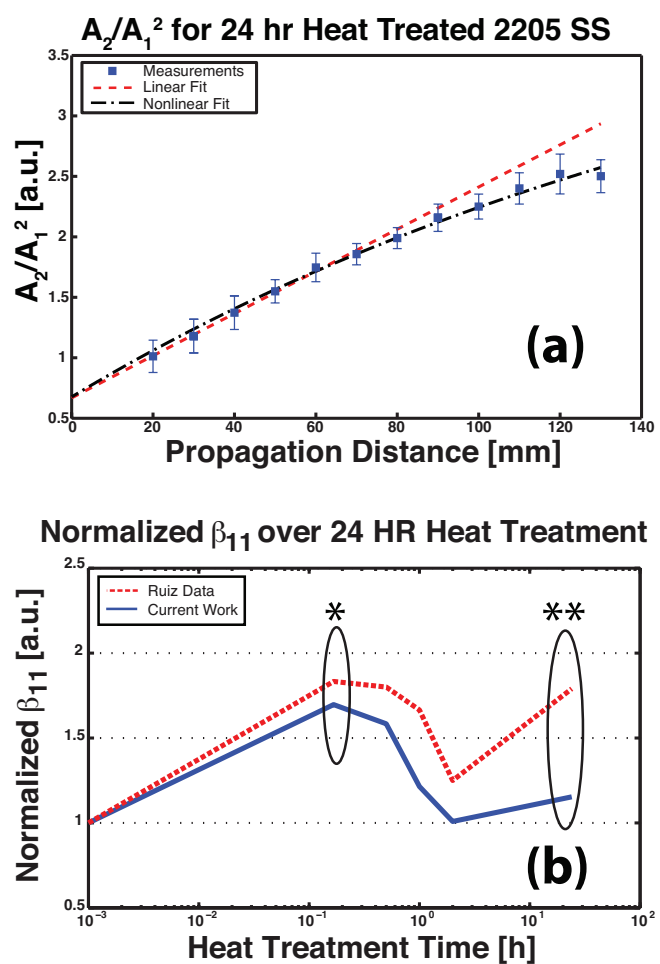

Figure 8: (a) shows a set of nonlinear measurements versus propagation distance for a 2205 SS sample heat treated over 24 hours. The red dotted line shows a linear fit to the "linear region" of the data, which is identified subjectively. The black dash-dotted line represents the results of the nonlinear fitting procedure. (b) shows the results of 2205 duplex stainless steel nonlinear parameter measurements as a function of heat treatment time for both the original analysis using a linear fitting approach (Ruiz et al) and the nonlinear fitting approach (current work). The data point labeled $(*)$ represents data collected at 10 minutes, and the data point labeled $\left({ }^{* *}\right)$ represents data collected at 24 hours. The $\beta_{11}$ values represented by each fitting procedure in (a) can clearly be seen as the last data point in (b).

The calculated $\beta_{11}$ values from both the linear (Ruiz data) and nonlinear (current work) methods are shown in figure $8(\mathrm{~b})$. The agreement of the general trends between the data sets confirms that the nonlinear fitting method of extracting the nonlinearity parameter produces results that are comparable to those found in the earlier work. Additionally, one source of confusion with the results obtained from the linear fitting technique was the rise of the nonlinear parameter value at 24 hours, labeled $(* *)$ on figure $8(\mathrm{~b})$, back to the heat treatment levels obtained at 10 minutes, labeled $\left(^{*}\right)$ on figure $8(\mathrm{~b})$, of treatment time. This trend was not observed in other material tests ${ }^{28,29,30}$, and the nonlinear fitting method proposed in the current work shows results more in line with those expected from experience and literature ${ }^{28}$. The accuracy and repeatability of the nonlinear fitting approach combined with the more realistic 

nonlinearity.

\section{Conclusions and Future Work}

In this work, it is postulated that, given a Gaussian line source approximation for the generation of a Rayleigh wave, physically accurate values for a relative measure of the nonlinearity parameter can be extracted by fitting the data to models accounting for diffraction, attenuation and transducer nonlinearity effects. By showing that the source function, which is a result of the wedge and transducer combination, can be accurately described as Gaussian in shape, we validate the use of this approach. Furthermore, the experiments show 510 that there exists a second harmonic component to the source function prior to generation effects from the sample material that is also Gaussian in shape, and that this effect must be accounted for in the model and the data. In order to fit the data taken from the air-coupled transducer setup to the proposed model, the use of a nonlinear least squares curve-fitting procedure is necessary because 515 many of the parameters required for the fit process are either difficult to measure or directly immeasurable. This process is done over multiple steps, the first fitting the fundamental frequency data, the second estimating the nonlinear source strength, and the third fitting the second harmonic attenuation and the nonlinear parameter, which is the ultimate desired result. This process is 520 shown for Al 2024 and 7075 samples, and the results are consistent with previous literature and physical expectations. Additionally, a borrowed data set for heat-treated 2205 duplex stainless steel is re-processed with the updated analytical model and it is found that the new values for the nonlinear parameter both match with the general trends from the previous results and amend them to agree with past literature and physical expectations of the treatment process.

While this work details a more refined process for calculating the nonlinearity parameter from experimental results, more experimental information could facilitate more accurate estimations of the fitting parameters. Measurement of the attenuation at the fundamental and second harmonics could serve to either confirm or directly substitute these values in the model, meaning fewer parameters to fit and thus more accuracy from the model. In addition, directly measuring the source strengths with the laser vibrometer before each data collection would allow for substitution of that information into the analytical formulation, leaving just the nonlinear parameter as the sole fitting variable.

${ }_{535}$ Another factor that is unknown in the procedure implemented in this work is the phase relationship between the fundamental and harmonic components of the source. This work treats these components as having the same phase, however phase differential could slightly alter the value of $\beta_{11}$ calculated from the curve fitting procedure. While the reasonableness of the results in this 540 paper validate the assumption about the relative phase, an experimental setup that could accurately and repeatably calculate this quantity would answer this question definitively. 
Additionally, the relationship of the output voltage from the air-coupled receiver to the received waveforms is more complicated than using axial solution to the fitting equation because the transducer receives a signal from an area distribution about the central measurement axis. This spatial weighting is a transducer property and will be necessary to understand moving forward. In theory, if the receiving transducer is accurately characterized and the transfer function known exactly, then the results of this procedure would be absolute 550 measures of the nonlinear parameter, which would be a very powerful tool in Rayleigh wave measurements for the NDE community.

\section{Acknowledgements}

We would like to acknowledge the U.S. Department of Energy Nuclear Energy University Program (NEUP) for funding this work. We would also like to thank Dr. Jennifer Michaels and her lab for the use of their Polytec LDV and positioning system for our laser measurements.

\section{Appendix A. Derivation of Diffraction Equations}

The following is a more detailed derivation of equations (7) and (8) according to the steps listed primarily in Shull et al ${ }^{19}$. First, we start with the quasilinear system given by equations (3) and (4):

$$
\begin{gathered}
\left(\frac{\partial}{\partial x}+\frac{1}{2 i k_{0}} \frac{\partial^{2}}{\partial y^{2}}+\alpha_{1}\right) v_{1}=0 \\
\left(\frac{\partial}{\partial x}+\frac{1}{4 i k_{0}} \frac{\partial^{2}}{\partial y^{2}}+\alpha_{2}\right) v_{2}=-\frac{\beta_{11} k_{0}}{2 c_{R}} v_{1}^{2}
\end{gathered}
$$

It is illustrative to note that these equations do not require the traveling wave to be plane. The lack of this condition stems from the derivation of the spectral equations from which the equations of motion (A.1) and (A.2) are formulated ${ }^{17}$. Now consider a source with the following conditions at $x=0$ :

$$
\begin{gathered}
v_{1}(0, y)=w(y) \\
v_{n}(0, y)=0, n>1
\end{gathered}
$$

This source need not be symmetric, and may be complex.

The integral solutions to equations (A.1) and (A.2) are formulated by employing a Green's function formulation, and are expressed in the following form:

$$
v_{1}(x, y)=\int_{-\infty}^{\infty} w\left(y^{\prime}\right) g_{1}\left(x, y \mid 0, y^{\prime}\right) \mathrm{d} y^{\prime}
$$




$$
v_{2}(x, y)=-\frac{\beta_{11} k_{0}}{2 c_{\mathrm{R}}} \int_{0}^{x} \int_{-\infty}^{\infty} v_{1}^{2}\left(x^{\prime}, y^{\prime}\right) g_{2}\left(x, y \mid x^{\prime}, y^{\prime}\right) \mathrm{d} x^{\prime} \mathrm{d} y^{\prime}
$$

where the Green's functions $g_{1}$ and $g_{2}$ are represented as:

$$
\begin{aligned}
& g_{1}\left(x, y \mid x^{\prime}, y^{\prime}\right)=\sqrt{\frac{k_{0}}{i 2 \pi\left(x-x^{\prime}\right)}} \exp \left(-\alpha_{1}\left(x-x^{\prime}\right)+\frac{i k_{0}\left(y-y^{\prime}\right)^{2}}{2\left(x-x^{\prime}\right)}\right) \\
& g_{2}\left(x, y \mid x^{\prime}, y^{\prime}\right)=\sqrt{\frac{2 k_{0}}{i 2 \pi\left(x-x^{\prime}\right)}} \exp \left(-\alpha_{2}\left(x-x^{\prime}\right)+\frac{i 2 k_{0}\left(y-y^{\prime}\right)^{2}}{2\left(x-x^{\prime}\right)}\right)
\end{aligned}
$$

Note that in the formulation of the Green's function for the velocity at the fundamental frequency $\left(g_{1}\right)$ the propagation is considered from $\left(0, y^{\prime}\right)$, which is the location of the source. However, in the case of Green's function for the second harmonic velocity $\left(g_{2}\right)$, the propagation is considered from all points on the surface $\left(x^{\prime}, y^{\prime}\right)$. This is due to the harmonic generation as the wave propagates and continually leaks energy from the fundamental frequency to its harmonics $^{17}$.

If we perform this integration and let $w(y)$ be defined as a Gaussian source function as in equation (6):

$$
w(y)=v_{0} e^{-(y / a)^{2}}
$$

then we have the necessary information to solve equation (A.5). To do this, we recast the integral into the form:

$$
\int_{-\infty}^{\infty} e^{-a y^{\prime 2}} e^{-2 b y^{\prime}} \mathrm{d} y^{\prime}=\sqrt{\frac{\pi}{a}} e^{\left(b^{2} / a\right)}
$$

After simplification, we arrive at the solution for $v_{1}$ given in equation (7). general approach as in the solution for $v_{1}$ but with the added complication of the second integral in $x^{\prime}$. An observation of solution given by equation (8) reveals two error functions. The first error function is associated with the particular solution and the second to the homogeneous solution to equation (4), where the particular solution describes the forced component and the homogeneous solution describes the free propagating component of the second harmonic wave ${ }^{16}$.

\section{References}

[1] J. Herrmann, J.-Y. Kim, L. J. Jacobs, J. Qu, J. W. Littles, M. F. Savage, Assessment of material damage in a nickel-base superalloy using nonlinear rayleigh surface waves, Journal of Applied Physics 99 (12) (2006) 124913124913. 
[2] S. V. Walker, J.-Y. Kim, J. Qu, L. J. Jacobs, Fatigue damage evaluation in a36 steel using nonlinear rayleigh surface waves, NDT \& E International 48 (0) (2012) $10-15$.

[12] A. Ruiz, P. B. Nagy, Diffraction correction for precision surface acoustic wave velocity measurements, The Journal of the Acoustical Society of America 112 (2002) 835.

[13] P. H. Rogers, A. L. V. Buren, An exact expression for the lommel-diffraction correction integral, The Journal of the Acoustical Society of America 55 (4) (1974) 724-728.

[14] G. S. Kino, Acoustic waves: devices, imaging, and analog signal processing, Vol. 107, Prentice-Hall Englewood Cliffs, NJ, 1987. 
[15] F. Ingenito, A. O. Williams, Calculation of second-harmonic generation in a piston beam, Journal of the Acoustical Society of America 49 (1971) 319-328.

[16] D. J. Shull, E. E. Kim, M. F. Hamilton, E. A. Zabolotskaya, Diffraction effects in nonlinear rayleigh wave beams, The Journal of the Acoustical Society of America 97 (4) (1995) 2126-2137.

[17] E. Zabolotskaya, Nonlinear propagation of plane and circular rayleigh waves in isotropic solids, The Journal of the Acoustical Society of America 91 (1992) 2569.

[18] D. C. Hurley, Nonlinear propagation of narrow-band rayleigh waves excited by a comb transducer, The Journal of the Acoustical Society of America $106(1999) 1782$.

[19] D. J. Shull, M. F. Hamilton, Y. A. Il'insky, E. A. Zabolotskaya, Harmonic generation in plane and cylindrical nonlinear rayleigh waves, The Journal of the Acoustical Society of America 94 (1) (1993) 418-427.

[20] R. Smith, R. Stern, R. Stephens, Third-order elastic moduli of polycrystalline metals from ultrasonic velocity measurements, The Journal of the Acoustical Society of America 40 (1966) 1002.

[21] C. Ramadas, A. Hood, I. Khan, K. Balasubramaniam, Effect of misalignment of air-coupled probes on $A_{o}$ lamb mode propagating in a metal plate, Ultrasonics 54 (5) (2014) 1401-1408.

[22] M. Deighton, A. Gillespie, R. Pike, R. Watkins, Mode conversion of rayleigh and lamb waves to compression waves at a metal-liquid interface, Ultrasonics 19 (6) (1981) $249-258$.

[23] D. Ensminger, L. J. Bond, Ultrasonics: fundamentals, technologies, and applications, CRC Press, 2011.

[24] L. E. Kinsler, A. R. Frey, A. B. Coppens, J. V. Sanders, Fundamentals of Acoustics, 4th Edition, John Wiley \& Sons, Hoboken, NJ, 1999.

[25] S. Thiele, J.-Y. Kim, J. Qu, L. J. Jacobs, Air-coupled detection of nonlinear rayleigh surface waves to assess material nonlinearity, Ultrasonics 54 (6) (2014) 1470-1475.

[26] W. T. Yost, J. H. Cantrell, The effects of artificial aging of aluminum 2024 on its nonlinearity parameter, in: D. O. Thompson, D. E. Chimenti (Eds.), Review of Progress in Quantitative Nondestructive Evaluation, Springer, 1993, pp. 2067-2073.

[27] P. Li, W. Yost, J. Cantrell, K. Salama, Dependence of acoustic nonlinearity parameter on second phase precipitates of aluminum alloys, in: IEEE 1985 Ultrasonics Symposium, 1985, pp. 1113-1115. 
[28] Y. Xiang, M. Deng, F.-Z. Xuan, C.-J. Liu, Experimental study of thermal degradation in ferritic cr-ni alloy steel plates using nonlinear lamb waves, NDT \& E International 44 (8) (2011) $768-774$.

[29] A. Viswanath, B. P. C. Rao, S. Mahadevan, P. Parameswaran, T. Jayakumar, B. Raj, Nondestructive assessment of tensile properties of cold worked AISI type 304 stainless steel using nonlinear ultrasonic technique, Journal of Materials Processing Technology 211 (3) (2011) 538 - 544.

[30] D. Barnard, G. Dace, O. Buck, Acoustic harmonic generation due to thermal embrittlement of inconel 718, Journal of Nondestructive Evaluation 16 (2) (1997) 67-75. 